TI 2012-039/3

Tinbergen Institute Discussion Paper
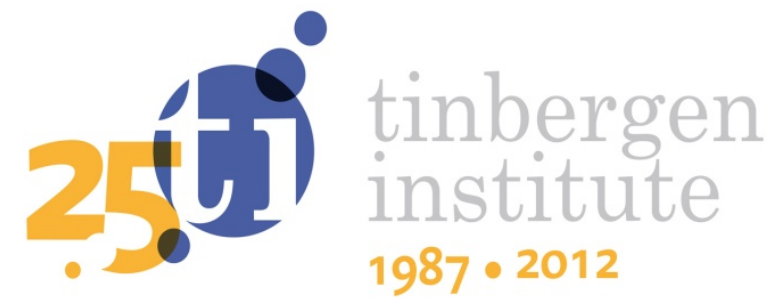

\title{
Effects of NCMS Coverage on Access to Care and Financial Protection in China
}

\author{
Zhiyuan Houa \\ Ellen Van de Poelb \\ Eddy Van Doorslaerb,c,d \\ Baorong Yua \\ Qingyue Menge
}

a Center for Health Management and Policy, Shandong University, Jinan, China.

$b$ Institute of Health Policy \& Management, Erasmus University Rotterdam, Netherlands

c Erasmus School of Economics, Erasmus University Rotterdam, Netherlands

a Tinbergen Institute, Rotterdam

e China Center for Health Development Studies, Peking University, China. 
Tinbergen Institute is the graduate school and research institute in economics of Erasmus University Rotterdam, the University of Amsterdam and VU University Amsterdam.

More TI discussion papers can be downloaded at http://www.tinbergen.nl

Tinbergen Institute has two locations:

Tinbergen Institute Amsterdam

Gustav Mahlerplein 117

1082 MS Amsterdam

The Netherlands

Tel.: +31(0)205251600

Tinbergen Institute Rotterdam

Burg. Oudlaan 50

3062 PA Rotterdam

The Netherlands

Tel.: +31(0)10 4088900

Fax: $+31(0) 104089031$

Duisenberg school of finance is a collaboration of the Dutch financial sector and universities, with the ambition to support innovative research and offer top quality academic education in core areas of finance.

DSF research papers can be downloaded at: http://www.dsf.nl/

Duisenberg school of finance

Gustav Mahlerplein 117

1082 MS Amsterdam

The Netherlands

Tel.: +31(0)20 5258579 


\title{
Effects of NCMS coverage on access to care and financial protection in China
}

Zhiyuan Hou ${ }^{a}$, Ellen Van de Poelb, Eddy Van Doorslaerb,c,d, Baorong

$$
\text { Yua, Qingyue Menge }
$$

\begin{abstract}
The introduction of the New Cooperative Medical Scheme in rural China is one of the largest health care reforms in the developing world since the millennium. The literature to date has mainly used the uneven rollout of NCMS across counties as a way of identifying its effects on access to care and financial protection. This study exploits the cross-county variation in NCMS generosity in 2006 and 2008 in Ningxia and Shandong province and adopts an instrumenting approach to estimate the effect of a continuous measure of coverage level. Our results confirm earlier findings of NCMS being effective in increasing access to care, but not increasing financial protection. In addition, we find that NCMS enrollees are sensitive to the incentives set in the NCMS design when choosing their provider, but also that providers seem to respond by increasing prices and/or providing more expensive care.
\end{abstract}

\section{Keywords}

Health insurance, access, financial protection, China

\section{Word count}

5488

\footnotetext{
${ }^{a}$ Center for Health Management and Policy, Shandong University, Jinan, China.

Email: fexplorer1019@163.com, bryu@sdu.edu.cn

${ }^{b}$ Institute of Health Policy \& Management, Erasmus University Rotterdam, Netherlands

Email: vandepoel@bmg.eur.nl

${ }^{c}$ Erasmus School of Economics, Erasmus University Rotterdam, Netherlands

Email: vandoorslaer@ese.eur.nl

${ }^{d}$ Tinbergen Institute, Rotterdam

${ }^{\mathrm{e}}$ China Center for Health Development Studies, Peking University, China

Email: qmeng@bjmu.edu.cn
} 


\section{INTRODUCTION}

A growing number of developing countries have recently initiated attempts to extend social health insurance coverage to the vulnerable groups in their populations using tax subsidies. One of the largest of such initiatives is the implementation, since 2003, of a health insurance scheme for the rural population, namely the New Cooperative Medical Scheme (NCMS) by the Chinese government. As a voluntary and heavily subsidized scheme, it has seen extremely rapid growth of coverage in comparison to most new schemes in developing countries (Hsiao and Shaw, 2007; Yip and Hsiao, 2008). By the end of $2010,96 \%$ of the rural population ( 840 million people) had been enrolled in NCMS (Ministry of Health, 2010). Generally, the NCMS is heavily oriented at inpatient care and, especially in the period before the 2009 Health Care Reform, was characterized by rather high copayments and coinsurance rates. Outpatient care was typically covered through Medical Savings Accounts, which involve no risk pooling. Although the central government has issued broad guidelines for the design of the scheme, the policy details and implementation responsibilities have been decentralized to the county level local governments (Brown et al., 2009). As a result, important scheme features, including the benefit package, the reimbursement level and payment methods, display considerable variation across counties and over time, which can be used to identify the impact of the scheme.

Many studies have recently sought to assess the impact of NCMS coverage on health care access and financial protection, mostly using the uneven rollout of NCMS 
across counties as a way of identifying its effects. ${ }^{1}$ Few studies so far have exploited the heterogeneity in scheme features to identify its effect. This study aims to add to the literature on the effects of NCMS, by using recent household data from Ningxia and Shandong province, complemented with administrative data. We limit our analysis to NCMS enrollees and exploit cross-county variation in NCMS characteristics to identify impact. We construct measures of average NCMS generosity using instrumenting methods, and evaluate effects of these on the probability of using care, the choice of provider and the amounts spent. In part, our results confirm earlier findings of NCMS being effective in increasing access to care, but not increasing financial protection. In addition, we find that NCMS enrollees are sensitive to the incentives set in the NCMS design when choosing their provider, but also that providers seem to respond by increasing prices and/or providing more expensive care.

The paper is organized as follows. First, section 2 discusses the literature on the effects of NCMS. Thereafter, as our empirical strategy is quite influenced by the nature of available data, section 3 first introduces a description of the study setting and data, and then, section 4 explains the methodological approach to identify the NCMS impact. Section 5 presents estimation results, followed by a summary of results and discussion.

${ }^{1}$ For a review of these studies see e.g. Wagstaff, Yip et al. (2009a); Yip and Hsiao (2009); Yip et al. (2009); You and Kobayashi (2009). 


\section{LITERATURE REVIEW}

Although cross-sectional studies present mixed results regarding the effects of NCMS on health care utilization (Hao et al., 2010; Yu et al., 2010; Zhou et al., 2011; Qian et al., 2009), those studies that address the problem of endogenous NCMS participation using panel data, generally do find that NCMS increases health care utilization. Wagstaff et al. (2009b) use a differences-in-differences analysis on a panel dataset from the 2003 and 2005 National Health Services Survey (NHSS) and conclude that NCMS has had an appreciable positive impact on the utilization of both outpatient (23\%) and inpatient services (27\%). Lei and Lin (2009), using the date of county-level NCMS inception as an instrumental variable for an individual's NCMS status (in data from the China Health and Nutrition Survey (CHNS)), find no effect of NCMS on utilization of formal medical service and a negative effect on the use of traditional Chinese doctors.

To examine the effect of NCMS on provider choice, Babiarz et al (2010) use data from a survey in 2004 and 2007 and find that enrolment in NCMS can increase the probability of seeking care at village clinics by $5 \%$ compared to Township Health Centers (THC) and higher level hospitals. Brown and Theoharides (2009) use cross sectional data from 2006 (from 25 counties) and find that hospital use increases with declining copayments and rising ceilings.

The evidence of NCMS offering financial protection is less compelling. Most studies find little or no impact of NCMS coverage on out-of-pocket expenditures (Lei and Lin, 2009; Wang et al., 2010; Liu and Tszegai, 2011) and some even report the 
curious finding of out-of-pocket spending to increase as a result of NCMS introduction (Wagstaff et al., 2009b; Zhou et al., 2009). Effects are also found not to be homogeneous across socioeconomic group. Wagstaff et al (2009b) and Yu et al (2010) find the increase in the use of inpatient care to be larger among rich than poor NCMS participants, and therefore also the increase in out-of-pocket spending to be larger among the richer enrollees. Using (panel) data from the CHNS, Liu and Tszegai (2011) find that NCMS does not reduce outpatient or inpatient expenditure, but increases the incidence of catastrophic expenditures in western regions.

Few studies so far have examined the heterogeneity of the NCMS' effects across scheme design and service packages. Using NCMS "policy bundles" characterized by level of health facility for outpatient services covered, and by outpatient reimbursement type, Babiarz et al. (2010) find that different "policy bundles" are not associated with different probabilities of medical care use and provider choices, but they do find more generous bundles to reduce the out-of-pocket medical spending. Wagstaff et al. (2009b) do identify quite some heterogeneity in the impact of NCMS across counties, but it remains unclear what drives this heterogeneity. In a more qualitative study, Yu et al. (2010) (using the same data as used in this paper) suggest that differences in health care utilization may be related to variation in NCMS policy design across counties, such as reimbursement rates and procedures, and outpatient reimbursement type. Zhong (2011) uses data from the 2008 pilot of the China Health and Retirement Longitudinal Study and reports that the likelihood of seeking inpatient treatment is negatively related to the coinsurance rate but positively to 
immediate reimbursement (relative to delayed reimbursement). However, this study does not have more detailed information on people's insurance coverage level (for inpatient and outpatient services).

\section{STUDY SETTING \& DATA}

We use the variation in NCMS design across six counties in two provinces, Ningxia and Shandong, and over two years (2006 and 2008) to estimate the effects of NCMS on access to care and financial protection. The survey and administrative data that we use were collected in three counties in Shandong province (Zhangqiu, Changle, and Dong'e) and Ningxia province (Yongning, Qingtongxia and Zhongning) for a study funded by the European Commission (RHINCAV) (Sun et al., 2009; Yu et al., 2010; Liu et al., 2012).

\subsection{NCMS design in the six study counties}

A detailed questionnaire was administered to NCMS officials to obtain information on the exact NCMS implementation details in each of the counties. Table 1 summarizes some of the relevant NCMS characteristics per county and survey wave. ${ }^{2}$ All six counties have started to implement NCMS since 2005 and were given considerable discretion in its design, i.e. benefit package, copayments, reimbursement rates and ceilings. NCMS coverage was already high in 2006 (at $88.3 \%$ ) and further increased to $94.6 \%$ of the population in 2008 . The bulk of NCMS funds are used for inpatient expenses, especially in Ningxia. While inpatient services

\footnotetext{
2 For a detailed discussion of the NCMS design, see e.g. Zhang et al (2010) and Yan et al (2011).
} 
are covered in all counties, the coverage degree varies across counties and over time, in terms of (flat rate) copayments, reimbursement rates and ceilings. Three counties in Shandong do not levy any copayments for hospitalizations in township health centers (THC), but Ningxia applies differentiated copayment levels for different levels of hospitals, ranging from $70 \mathrm{RMB}$ for $\mathrm{THC}$ to $500 \mathrm{RMB}$ for hospitals above county level. Reimbursement rates also vary considerably across counties, and with level of spending. For example, Figure 1, showing the non-linear reimbursement scheme for inpatient care at township health centers in Qingtongxia County in 2006, illustrates that each hospitalization requires a copayment of $150 \mathrm{RMB}$ and that the NCMS reimbursement rate increases with the amount of total spending until the cap of $10000 \mathrm{RMB}$ is reached. In Shandong, only outpatient visits in village clinics and THC are reimbursed, while in Ningxia the lower level village clinics are excluded from reimbursement. In Zhangqiu and Changle counties (Shandong), expenditures for outpatient care are covered on a risk pooling basis (Social Pooling Account (SPA)), while other counties have set up household Medical Savings Accounts (MSA). This means that households save a fixed amount per capita per year into the MSA to pay for outpatient visits when necessary. This is an earmarked savings account rather than a risk pooling mechanism, but the MSA can be shared among household members and rolled over to the next year so that funds can be accumulated. Once the MSA is exhausted there is generally no further reimbursement for outpatient services available. In Dong'e (Shandong) and Zhongning (Ningxia), however, in 2008 the SPA was applied to cover outpatient expenditures of families that had exhausted 
their MSA.

Table 1 and Figure 1 should be inserted about here.

\subsection{Data}

The household data derive from a repeated cross-section household survey conducted in Shandong and Ningxia Province in 2006 and 2008. In 2006, within each county, 18 villages spread across 3 townships were selected to participate in the survey. About 60 households were randomly selected per village in Shandong, and about 50 in Ningxia, resulting in a total sample of 6,137 households $(22,636$ individuals). In 2008, a slightly smaller sample of households (30 in both provinces) was drawn from the same villages, leading to a sample size of 3,288 households (13,058 individuals). In our analysis, we only use adult individuals aged 16 or older and enrolled with NCMS, which amounts to a total sample size of 26,310 individuals $(16,294$ in 2006 and 10,016 in 2008). We use the sample of non-NCMS members (2528 individuals) only to do a (placebo) test for the absence of any effect of NCMS coverage generosity on health care $u .^{3}$ Note that the data is a panel at the village level, but not at the individual level.

The outcomes of interest in this study relate to health care utilization and financial protection. For utilization, we have indicators for whether individuals used inpatient (last year) or outpatient care (last four weeks), indicators for the choice of in- and outpatient facility (for the first visit in the last year/4 weeks) and for the

\footnotetext{
3 The sample of non-NCMS members that have used inpatient or outpatient care is too small (206 and 105 individuals respectively) to allow for separate analysis.
} 
length of stay for inpatient care (for the first visit in the last year). Regarding financial protection, we use variables capturing the total and out-of-pocket (OOP) spending by individuals and the share of OOP spending of the total health care spending (for the same first visit in the relevant recall period), and the proportion of health expenditure in household expenditures. All spending data are deflated to 2006 values using province specific Consumer Price Indices. ${ }^{4}$

Our models also include a vector of individual level covariates on demographics (age and sex, marital status, household size), socioeconomic condition (education, occupation, log income ${ }^{5}$ ) and enrolment with other insurance schemes than NCMS. ${ }^{6}$ To capture variation in need for care across individuals we also include a set of variables related to individuals' health status. In models for outpatient care we include information on sickness in the four weeks preceding the survey, self-perceived severity of illness and rest days due to sickness. Models on the probability of inpatient care include variables related to chronic diseases and disease severity, while models on length of stay and financial protection also have information on diagnosed diseases and whether or not the hospitalization involved

\footnotetext{
${ }^{4}$ Consumer Price Indices are taken from China Statistical Yearbook 2008 \& 2009.

${ }^{5}$ Household income is calculated by summing all market earnings across the household as well as the total value of all other non-market goods and services produced within the household. Totals are deflated to 2006 values using province specific CPI and divided by household size to obtain per capita estimates.

${ }^{6}$ We do not have details on this supplemental health insurance cover, but the data suggest this is mostly private, commercial insurance as coverage is concentrated among the rich and higher educated. Therefore we have excluded individuals with additional coverage from the analysis on financial protection.
} 
surgery. ${ }^{7}$ The International Classification of Diseases (ICD 10) (World Health Organization, 2007) is used to classify respondents' reported disease symptoms into about 20 main categories, which were further reduced to about 5 categories to ensure sufficient observations in each category. Appendix 1 provides more detail and some descriptive information of all covariates used.

Next to household level data, we also have access to aggregate data from the NCMS claims offices in each of the counties on the total amount of insurance claims and reimbursements made for the entire NCMS population within that county.

\section{METHODS}

We aim to identify the impact of NCMS on access to care and financial protection by exploiting the variation in NCMS design across counties. This section first describes the constructed measures of NCMS generosity and the models used to estimate their effect.

\subsection{Calculation of NCMS coverage degree}

The scheme's degree of coverage for a specific service depends on three characteristics: (i) the copayment, (ii) the reimbursement rate, and (iii) the ceiling. For outpatient care and for counties with SPA, (ii) and (iii) are typically constant ${ }^{8}$ and (i) does not apply. For inpatient care, (i) and (ii) vary with the provider chosen, and (ii) also depends on the total costs of the hospitalization (see Figure 1 for an example).

\footnotetext{
7 The set of ill-health variables is not constant across all models because some survey questions are specific to the section on in- or outpatient care.

${ }^{8}$ The reimbursement for outpatient care only varies with providers and services in Zhangqiu County, and only counties in Shandong apply a ceiling for outpatient expenditures (Table 1).
} 
Given this variation in coverage of inpatient care, and the limited number of county/years observations, we combine all three dimensions of cover into one measure to capture the NCMS coverage degree for inpatient care across counties/years.

Calculating average reimbursement rates (ARR) based on households' expenditures and NCMS reimbursements as recorded in the household data could be affected by individuals' behavioral responses to differences in NCMS, especially for inpatient care for which the reimbursement rate varies with the total costs of care. As a result, the coverage rate may be co-determined by the (average) utilization to be explained. Average reimbursement rates calculated from the claims data are less likely to be affected directly by the behavioral response of households in the survey, but to the extent that the sample reflects the behavior of the entire relevant population group the endogeneity problem does remain. To deal with this potential endogeneity problem, we adopt two different approaches. The aim is therefore to obtain a summary measure of the extent to which NCMS insured are covered, which is not (or at least less) dependent on the utilization decisions of the relevant populations.

In a first approach, we use an instrumental variables (IV) method to estimate measures of NCMS coverage per county. We use official reimbursement rates, copayments and ceilings, as shown in Table 1, as instruments for the average coverage rates calculated from the NCMS claims data. These instruments are exogenous and only affect health care use through their effect on NCMS coverage, 
thereby satisfying the two necessary conditions for valid instruments (Wooldridge, 2002). First stage regressions are run separately for all types of health care providers, and weighted averages of predictions from these models are then used as generosity measures (predicted ARR). ${ }^{9}$ While this approach should deal with the endogeneity problem, the small number of county-level observations (12) limits the power to identify the contributions of scheme parameters in the first stage regressions.

Therefore, in a second approach we construct a measure of NCMS coverage, independent of the county-specific utilization behavior, using a standardization approach. First we calculate for each individual in the sample, the proportion of hospitalization expenditures that $s /$ he would have been reimbursed under each of the 12 (6 counties times 2 waves) NCMS schedules. For each county and wave, we then average these estimated proportions across all individuals to obtain the average reimbursement rate (standardized ARR). ${ }^{10}$ In effect, it indicates what NCMS reimbursement would have been had the entire sample lived in the relevant county and been subjected to the reimbursement rules of the relevant year. Because each county's coverage rate is based on the health care use pattern of the entire sample, variation in this rate only reflects differences in the generosity of the NCMS scheme. The approach does assume that there is no variation across counties in the type of drugs and services eligible for reimbursement. ${ }^{11}$

\footnotetext{
${ }^{9}$ Weights are calculated as frequency of visits with the specific health care provider in the county (calculated from claims data).

10 This approach is inspired by Contoyannis et al. (2005).

${ }^{11}$ While each county can define its list of covered drugs and services in accordance with national and provincial guidelines, in practice, the uncovered services are very similar across counties.
} 
For outpatient care, most of the cross-county variation in NCMS designs derives from the type of account (SPA or MSA). Among counties with SPA, the variation in reimbursement rates is rather limited. We therefore only investigate whether the effects of SPA type schemes differ from MSA.

\subsection{Model estimation}

Our strategy for identifying the effect of coverage on health care access, choice and financial protection basically relies on a pooled regression analysis with predicted $A R R$ and standardized $A R R$ as our main variables of interests. The binary outcomes are assumed to be generated as follows:

$$
y_{i t}=f\left(\lambda_{t}+\alpha_{v}+x_{c t} \beta+z_{i t} \gamma+\varepsilon_{i t}>0\right)
$$

where $f($.$) is the indicator function and y_{i t}$ indicates health care of individual $\mathrm{i}$ at time t. The model includes a time effect $\lambda_{t}$, which captures the trend in health care use between the two waves that is common across all individuals; a full set of village fixed effects $\alpha_{v}$ which absorb time invariant geographic differences; and a vector of time varying county specific variables, $x_{c t}$, which includes the NCMS coverage rate (predicted ARR or standardized ARR) and county level GDP per capita (see second row in Table 1) of county $c$ at time $t$; and a set of time-varying, individual specific covariates $z_{i t}$ to take account of the differences in observable determinants of health care use. For the dichotomous health care use outcomes, and the choice of medical facility, we assume the error term $\varepsilon_{i t}$ is standard logistically distributed such that (1) is a conditional logit model. When modeling length of hospital stay, we replace $f($.$) with the identity function and assume that the error is normally$ 
distributed such that (1) reduces to a linear village fixed effects model.

Outcomes related to financial protection $\left(y^{\prime}{ }_{i t}\right)$ are modeled using Generalized Linear Models (GLM), which are well suited to deal with skewed outcomes and avoid retransformation problems (Mihaylova et al., 2011; Manning and Mullahy, 2001). The central structure of a GLM is an exponential conditional mean:

$$
g\left\{E\left(y^{\prime}{ }_{i t}\right)\right\}=\lambda_{t}+\alpha_{v}+x_{c t} \beta+z_{i t} \gamma, \quad y^{\prime}{ }_{i t} \sim F
$$

with $g($.$) representing the link function and F($.$) the distributional family. To$ model total and out of pocket expenditures on health care, we used Box-Cox tests and Modified Park Tests to select, respectively, the link function and the family of GLM models that best suits our data (Manning and Mullahy, 2001). The preferred GLM specification was one with log link and gamma family. The proportion of out of pocket spending in total spending is modeled by a GLM with the logit link and the binomial family (Papke and Wooldridge, 1996; Wooldridge, 2001).

Despite taking account of additive village effects in all models, we nonetheless adjust standard errors for clustering at the county level (see e.g. Bertrand et al., 2004). This clustering allows for the possibility of serially correlated county-level shocks (Angrist and Pischke, 2009, p.319), but inflates standard errors. Given the limited number of counties (6), a further consequence of this clustering is that we have limited degrees of freedom. We have therefore checked robustness of results to clustering on the village level, and confirmed that inference presented in this paper is conservative. 


\section{RESULTS}

\subsection{Descriptive statistics}

Table 2 shows summary statistics for all dependent variables, and Appendix 1 for all independent variables. The average probability of NCMS enrollees seeking in- and outpatient care is $5.4 \%$ and $9.9 \%$, and the average spending per admission and visit is 4300 and 390 RMB respectively.

Table 2 should be inserted here.

\subsection{Effects of NCMS on utilization of care}

Table 3 shows selected estimation results for models explaining the probability of using both in- and outpatient care. For parsimony, marginal effects are only shown for a selected set of covariates, but all models include covariates listed in Appendix 1. The first two columns show marginal effects from a conditional logit model of the probability of a hospitalization in the last year, with a positive and significant coefficient for both measures of NCMS coverage. A one percentage point increase in NCMS coverage raises the probability of using inpatient care by $0.003-0.004$, which corresponds to an increase of about $7 \%$ compared to the overall sample probability (0.05). An increase of 40 percentage points in NCMS coverage, which is equal to the range in NCMS coverage rate in the sample (both in predicted ARR and standardized $A R R$ ), would raise the admission probability by 0.16 . The presence of other, mostly private health insurance cover raises the probability of using inpatient care by 0.06 , or the equivalent of a 15 percentage points rise in NCMS coverage. Using a sort of 
placebo test, we found no significant effect of county NCMS coverage on the probability of using inpatient care among non-NCMS members.

Table 3 should be inserted here.

The third column of Table 3 shows estimates for selected covariates on the probability of using inpatient care at county hospital or higher level (1), as compared to THCs (0). ${ }^{12}$ For this model, we use the ratio of the weighted average reimbursement rates for county and higher level hospitals to the reimbursement rate for THCs and only use the much smaller sample of those hospitalized in the last year (1036 observations). ${ }^{13}$ Because of the limited number of users of some facilities, especially at higher level, we can only show results for the predicted $A R R$, not for the standardized $A R R$. A one unit increase in the ratio of average reimbursement rates raises the probability of seeking care at a county hospital or higher level by 0.9 . The maximum variation in this ratio in the sample is $0.48^{14}$, which corresponds to an increase of 0.43 in the probability of using higher level care, equivalent to a $54 \%$ increase compared to the baseline mean (0.79).

The fourth and fifth columns show coefficients from an OLS model for (the log of)

\footnotetext{
${ }^{12}$ For outpatient care, the variation in the type of facilities that qualify for reimbursement varies between provinces. In Ningxia, only outpatient visits at the county hospital or higher can be reimbursed, while in Shandong only visits at the village clinics qualify for NCMS reimbursement. The effects of this difference in NCMS design can therefore not be distinguished from the province effect.

${ }^{13}$ Sample size is reduced further (375 observations) because the conditional logit (with village fixed effects) only uses those villages for which there is variation in the choice of IP provider - on average there are only 10 users per village. Results are robust to replacing village fixed effects by town fixed effects (1324 observations), and significance is increased.

14 This is the difference in reimbursement ratios between Qingtongxia in 2006 (0.82) and Zhangqiu in 2006 (0.34).
} 
length of inpatient stay. The effect of NCMS coverage is positive but not significant for either measure of coverage. A surgical treatment, admission at a higher level facility, and having other types of health insurance cover are all found to lengthen the hospital stay.

The last column of Table 3 displays estimation results for utilization of outpatient care and reveals that a system of risk pooling leads to a significantly higher probability of an outpatient visit (by 6 percentage points) than a system of Medical Saving Accounts, or a combination of both. Interestingly, having other health insurance cover is found to lower the probability of an outpatient visit, which may be an indication that privately insured individuals are more inclined to seek (covered) higher level care.

\subsection{Effects of NCMS on financial protection}

Having established the effects on utilization patterns, we want to verify whether, on balance, a higher degree of NCMS coverage provides covered individuals with greater financial protection. Results for NCMS effects on financial protection for in- and outpatient care are presented in Table 4 and 5 respectively. Note that these models are only based on the sample of individuals that have used the relevant type of care and do not have supplemental insurance.

Table 4 and 5 should be inserted here.

Higher NCMS average reimbursement rates for inpatient care do lead to increased total spending for an inpatient visit (first and second column in Table 4), while they only weakly affect the out of pocket payments made by individuals (third 
and fourth column). A one percentage point increase in the standardized ARR (predicted ARR) leads to a $33 \mathrm{RMB}$ (24 RMB) increase in total spending, which is about a $0.8 \%(0.6 \%)$ rise compared to the sample average of $4259 \mathrm{RMB}$. As expected, hospitalizations in more specialized facilities and involving surgery are found to substantially raise the total cost, as well as the amount of OOP spending. The fifth and sixth columns show effects of covariates on the share of OOP spending in total spending, and illustrate that a one percentage point increase in NCMS coverage leads to a decrease in the OOP share of 0.003 (the average share is 0.84 ), although the effect is barely significant. An admission to a higher level facility is associated with a higher OOP share, a longer stay with a lower share. The latter finding is probably related to the non-linearity of the reimbursement rate: typically NCMS schemes become more generous at higher levels of spending.

Estimated effects of NCMS coverage on financial protection from expenditures on outpatient care are presented in Table 5. We do not find significant effects of SPA on total spending per visit or on out-of-pocket spending per visit (first and second column), but we do find SPA to substantially reduce the share of OOP spending per visit (by 0.063 ) as compared to MSA (the average share is 0.93 ) (third column).

Finally, Table 6 shows results of NCMS coverage on health care spending at the household level. It suggests that a NCMS scheme with SPA for outpatient care increases a households' budget share spent on outpatient care (by 0.011 -see first column), while a more generous reimbursement scheme for inpatient care has no significant effect on the budget share of inpatient care (second and third column). 
These results reflect the combination of more generous NCMS schemes increasing health care use while not lowering the cost per visit, and can be interpreted as NCMS not delivering a high degree of financial protection.

Table 6 should be inserted here.

\section{DISCUSSION AND CONCLUSIONS}

The introduction of the NCMS in rural China represents one of the largest, and most impressive, health care reforms in the developing world since the millennium. The literature to date has mainly used the uneven rollout of NCMS across counties as a way of identifying its effects on access to care and financial protection. This study uses a different approach. It exploits the cross-county variation in NCMS generosity in two recent years in two provinces and adopts an instrumenting approach to estimate the access and protection effects of a continuous measure of coverage level.

We find clear evidence that adoption of a more generous scheme raises the probability of using both inpatient and outpatient care. Not surprisingly, the SPA system for outpatient care is more effective in improving access than the system of household MSA. Given the well-documented low access to medical care in rural China (Center for Health Statistics and Information, 2004, 2009), and the considerable copayments still required under the current NCMS schemes in place (especially for inpatient care), discretionary use of care is likely to be limited and the observed effect on use is therefore likely to mostly reflect better access to needed medical care. We also find evidence of variation in NCMS coverage affecting the 
choice of provider for inpatient care: enrollees are more likely to choose the provider with the higher reimbursement.

Our results indicate that NCMS also extends financial protection, as NCMS reduces the share of OOP spending for an inpatient and outpatient visit, but these effects are relatively small. More worrisome is that we find higher NCMS cover to raise total spending, per outpatient visit as well as per hospitalization, and to increase the OOP spending per inpatient stay. As this effect is larger for total than for OOP spending (even three times as large for inpatient care), this very much suggests that users are using - or providers are providing - more expensive care. We cannot ascertain whether and to what extent this more expensive use of care is more or less appropriate or of higher or lower quality, but given that providers are typically paid on a fee-for-service basis in these counties, they do have an incentive to respond to greater coverage by providing more expensive - and potentially unnecessary -- care (Yip and Hsiao, 2008; Hu et al., 2008; Wang et al., 2011). This may imply that the increased health spending has greater effect on provider welfare rather than on patient welfare.

In sum, these results do indicate some degree of success for NCMS in providing better access and financial protection for China's rural population, but issues of supply-side moral hazard remain a real threat to the system. This is consistent with recent results from a trend study using the National Health Services Survey, which shows that hospital admissions in rural areas had increased from $3.4 \%$ in 2003 to $6.8 \%$ in 2008 but that the proportion of households incurring catastrophic health spending 
levels also increased from 13.6\% in 2003 to $15.1 \%$ in 2008 (Meng et al., 2012).

In drawing these conclusions, there are some limitations to be kept in mind. First, while the data we use are very rich in many respects, our NCMS effects identification relies on information for six counties at two points in time, and hence has limited power to identify effects from variation in NCMS design. This may be the reason why we do not find significant heterogeneity in effects by poverty status. Second, as the data are only a panel at the village level, not at the individual level, we can only control for unobserved time-invariant heterogeneity at the village level. Third, the variation in NCMS design and generosity across counties may not be entirely exogenous and may well depend on factors also influencing individuals' health care seeking behavior across counties (e.g. quality of care). However, given that we control for a large set of individual and household level characteristics, village fixed effects and county GDP per capita, the problem of omitted variable bias is unlikely to be large. In spite of these limitations, we feel comfortable to conclude that in the first phase the expanded degree of coverage offered by NCMS in China has contributed to improved access to services to the rural populations in Ningxia and Shandong, but does not (yet) offer them sufficient protection against the risk of incurring catastrophic levels of health expenditure, given the high share of costs still borne by NCMS enrollees.

This may change in the near future. In 2009 China unveiled its health-care reform plan towards achieving comprehensive universal health coverage by 2020 (Central Committee of the Communist Party, 2009). The rapid rise in government subsidies to 
NCMS, a three-fold increase from 80 RMB per enrollee in 2008 to 240 RMB in 2012, has substantially reduced the patient copayment share. Moreover, more than $\mathbf{7 0}$ percent of counties have realigned their provider payment methods from fee-for-service to more prospective payment methods (Ministry of Health, 2012). Preliminary evidence suggests that financial protection has increased following these reforms (Yip et al., 2010; Meng et al., 2012) but further rigorous evaluation is warranted to assess whether China is on a sustainable track towards a more accessible system of health care coverage.

\section{ACKNOWLEDGEMENTS}

The data were collected in the project "Bringing health care to the vulnerable: developing equitable and sustainable rural health insurance in China and Vietnam (RHINCAV)" that was funded by the European Commission (and coordinated by the Liverpool School of Tropical Medicine (Specific Targeted Research Project nr 015305). Part of this work was also funded through the EU-FP7 research grant (HEALTH-F2-2009-223166-HEFPA) on "Health Equity and Financial Protection in Asia". Zhiyuan Hou acknowledges support from the China Scholarship Council for a scholarly visit to Erasmus University. We are grateful to all participants in the six study counties, and to officials from the Ministry of Health of China for support to the organization of the study. We also thank Winnie Yip, Owen O'Donnell and Adam Wagstaff for useful suggestions and comments and colleagues and students from Shandong and Fudan Universities for information on data issues. We declare that we have no conflict of interest. 


\section{TABLES \& FIGURES}

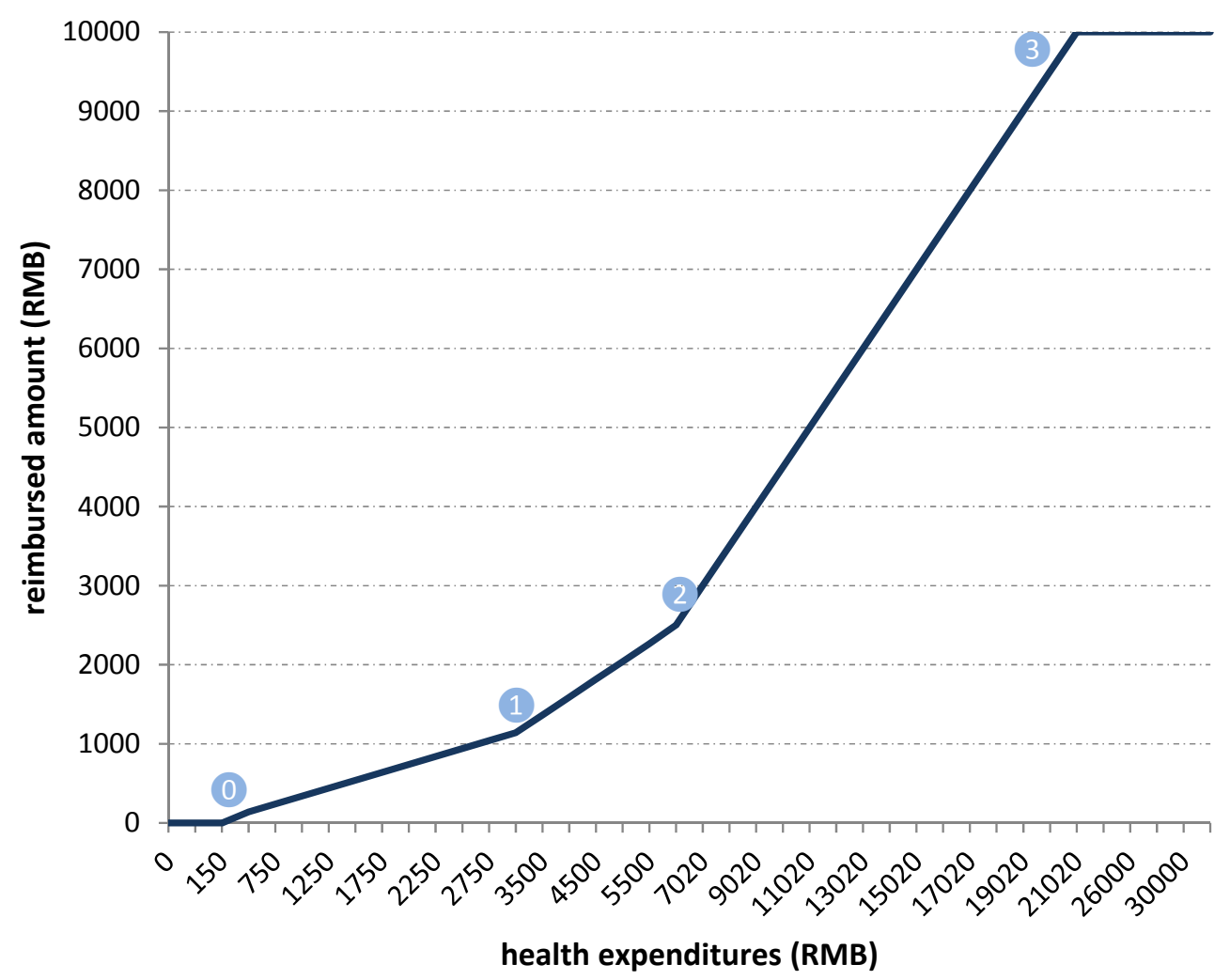

Figure 1: Graphical representation of the NCMS non-linear reimbursement schedule for inpatient care at township health centers at Qingtongxia County (Ningxia) in 2006

Notes: Reimbursement schedule applies to each visit. Fixed copayment of 150 RMB ( (0), 40\% reimbursement rate for part between 150 RMB and 3000 RMB (0)-1), 45\% for part between 3000 RMB and 6000 RMB (1-2), 50\% for part over 6000 RMB (2)-3). Reimbursement capped at 10000 RMB. The scale of the $\mathrm{X}$-axis varies across intervals of health expenditures. 


\begin{tabular}{|c|c|c|c|c|c|c|c|c|c|c|c|c|}
\hline & \multicolumn{6}{|c|}{ Shandong } & \multicolumn{6}{|c|}{ Ningxia } \\
\hline & \multicolumn{3}{|c|}{2006} & \multicolumn{3}{|c|}{2008} & \multicolumn{3}{|c|}{2006} & \multicolumn{3}{|c|}{2008} \\
\hline & Zhangqiu & Changle & Dong'e & Zhangqiu & Changle & Dong'e & Yongning & Qingtongxia & Zhongning & Yongning & Qingtongxia & Zhongning \\
\hline Rural population & 84.0 & 46.8 & 31.1 & 82.5 & 48.4 & 33.1 & 14.6 & 17.9 & 24.2 & 14.7 & 18.8 & 22.9 \\
\hline GDP per capita (RMB) & 26696 & 12093 & 12602 & 29039 & 15651 & 16815 & 11476 & 22443 & 11217 & 14701 & 24645 & 13059 \\
\hline NCMS enrolment rate (\%) & 89.9 & 90.6 & 93.5 & 94.9 & 97.7 & 96.8 & 91.6 & 90.8 & 73.4 & 96.3 & 96.8 & 86.6 \\
\hline NCMS budget per capita (RMB) & 40 & 40 & 40 & 70 & 75 & 70 & 50 & 50 & 50 & 100 & 100 & 100 \\
\hline Outpatient share of total fund (\%) & 47.44 & 22.13 & 30.8 & 52.72 & 18.58 & 20.67 & 12.98 & 16.83 & 19.95 & 7.18 & 11.27 & 9.87 \\
\hline \multicolumn{13}{|l|}{ Copayment per stay (RMB) } \\
\hline Township health center & 0 & 0 & 0 & 0 & 0 & 0 & 100 & 150 & 120 & 100 & 100 & 70 \\
\hline County hospital & 1000 & 0 & 0 & 1000 & 0 & 0 & 200 & 250 & 300 & 200 & 250 & 200 \\
\hline Higher level facility & N/A & 0 & 0 & 1000 & 3000 & 3000 & 500 & 500 & 500 & 500 & 500 & 500 \\
\hline \multicolumn{13}{|l|}{ Official inpatient reimbursement rate (\%)* } \\
\hline Township health center & 20 & 40 & 25 & 25 & 50 & 55 & 50 & 40 & 40 & 70 & 75 & 80 \\
\hline County hospital & 20 & 10 & 15 & 25 & 20 & 40 & 45 & 35 & 35 & 70 & 65 & 65 \\
\hline Higher level facility & 0 & 5 & 7.5 & 20 & 20 & 30 & 15 & 15 & 15 & 25 & 25 & 25 \\
\hline Ceiling for total inpatient reimbursement per & 14000 & 5000 & 5000 & 30000 & 20000 & 20000 & 10000 & 10000 & 10000 & 20000 & 15000 & 20000 \\
\hline Average inpatient reimbursement rate (\%) & 8 & 16 & 16 & 16 & 24 & 35 & 33 & 26 & 25 & 50 & 47 & 50 \\
\hline Outpatient reimbursement type & SPA & SPA & MSA & SPA & SPA & SPA/MSA & MSA & MSA & MSA & MSA & MSA & SPA/MSA \\
\hline Official outpatient reimbursement rate (\%) & $10-15$ & 15 & $6 \mathrm{RMB}$ & $20-40$ & 25 & 25 & $8 \mathrm{RMB}$ & $9 \mathrm{RMB}$ & $10 \mathrm{RMB}$ & $11 \mathrm{RMB}$ & $11 \mathrm{RMB}$ & $20 \%$ for \\
\hline Ceiling for total outpatient reimbursement per & & 200 & N/A & & 200 & 100 & N/A & N/A & N/A & N/A & $\mathrm{N} / \mathrm{A}$ & N/A \\
\hline Village clinic & 80 & & & 80 & & & & & & & & \\
\hline Township health center & 500 & & & 600 & & & & & & & & \\
\hline
\end{tabular}

Table 1. Details on NCMS design in 6 counties, 2006 and 2008.

Notes: Official reimbursement rates vary with the amount of health expenditures. The table shows reimbursement rates that would apply at the median level of spending in the total sample (2000 RMB). 


\begin{tabular}{lcc}
\hline Dependent variables & Observations & Mean (SD) \\
\hline Health care use & 26310 & 0.054 \\
Inpatient spell last 12 months (1/0) & 1411 & 0.210 \\
Inpatient at THC among users (1/0) & 1411 & 0.790 \\
Inpatient at county hospital or higher among users (1/0) & 1402 & $12.03(17.28)$ \\
Length of stay (days) & 26310 \\
Outpatient visit last 4 weeks (1/0) & 1935 & 0.099 \\
Outpatient visit at village clinic among users (1/0) & 1935 \\
Outpatient visit at THC or higher among users (1/0) & 0.740 \\
Individual level financial protection & 1377 \\
Total expenditures per inpatient spell (RMB) & 1377 \\
Out-of-pocket expenditures per inpatient spell (RMB) & 1378 \\
Out-of-pocket share of total expenditures per inpatient spell (0-1) & $37259(8216)$ \\
Total expenditures per outpatient visit (RMB) & 2422 \\
Out-of-pocket expenditures per outpatient visit (RMB) & 2422 & $390(7673)$ \\
Out-of-pocket share of total expenditures per outpatient visit (0-1) & $362(2091)$ \\
Household level financial protection & 2443 & $0.928(0.155)$ \\
Proportion of outpatient expenditure (last 4 weeks) in household net expenditure (0-1) & 5685 & $0.176)$ \\
Proportion of inpatient expenditure (last year) in household net expenditure (0-1) & 5685 & $0.099(0.958)$ \\
\hline
\end{tabular}

Table 2. Summary statistics of health care use and expenditure variables.

Notes: Standard errors are shown between brackets for continuous variables. 


\begin{tabular}{|c|c|c|c|c|c|c|}
\hline & $\begin{array}{r}\text { Probability of } \\
\qquad(1\end{array}$ & ospitalization & $\begin{array}{c}\text { County } \\
\text { hospital or } \\
\text { higher }(1 / 0)\end{array}$ & Log length $c$ & ay (days) & $\begin{array}{c}\text { Probability of } \\
\text { outpatient visit } \\
(1 / 0)\end{array}$ \\
\hline & \multicolumn{3}{|c|}{ Marginal effects from logit } & \multicolumn{2}{|c|}{ OLS coefficients } & Marginal offocts \\
\hline & $\begin{array}{l}\text { Standardized } \\
\text { ARR }\end{array}$ & Predicted ARR & Predicted ARR & $\begin{array}{l}\text { Standardized } \\
\text { ARR }\end{array}$ & $\begin{array}{l}\text { Predicted } \\
\text { ARR }\end{array}$ & from logit \\
\hline Log income & 0.001 & 0.001 & 0.005 & 0.016 & 0.016 & 0.007 \\
\hline Surgery & & & & $0.270^{* *}$ & $0.270^{* *}$ & \\
\hline $\begin{array}{l}\text { Admission at county hospital(reference } \\
\text { THC) }\end{array}$ & & & & $0.253^{* *}$ & $0.253^{* *}$ & \\
\hline Admission at higher hospital & & & & $0.529 * * *$ & $0.530 * * *$ & \\
\hline NCMS coverage (\%) & $0.004 * * *$ & $0.003 * * *$ & & 0.004 & 0.004 & \\
\hline $\begin{array}{l}\text { NCMS coverage for county hospitals and } \\
\text { higher/NCMS coverage for THCs }\end{array}$ & & & $0.943 * * *$ & & & \\
\hline SPA (reference MSA) & & & & & & $0.060^{*}$ \\
\hline Other health insurance & $0.059^{* *}$ & $0.051^{* *}$ & 0.05 & $0.120^{* *}$ & $0.119 * *$ & 0.024 \\
\hline Year 2008 & -0.016 & -0.004 & 0.061 & 0.051 & 0.057 & 0.133 \\
\hline Observations & 26296 & 26296 & 1036 & 1398 & 1398 & 26296 \\
\hline
\end{tabular}

Table 3. Regression results for utilization of inpatient and outpatient care

Notes: All models include covariates as shown in Appendix 1 and village fixed effects. Standard errors adjusted for clustering at county level. Significance level: $* * *$ at $1 \%, * *$ at $5 \%, *$ at $10 \%$. 


\begin{tabular}{|c|c|c|c|c|c|c|}
\hline & \multicolumn{2}{|c|}{ Total spending per stay (RMB) } & \multicolumn{2}{|c|}{$\begin{array}{l}\text { Out-of-pocket spending per stay } \\
\qquad(\mathrm{RMB})\end{array}$} & \multicolumn{2}{|c|}{ Out-of-pocket share (0-1) } \\
\hline & Standardized ARR & Predicted ARR & Standardized ARR & Predicted ARR & $\begin{array}{l}\text { Standardized } \\
\text { ARR }\end{array}$ & Predicted ARR \\
\hline Log income & 191.8 & 190.9 & 156.5 & 156.2 & 0.005 & 0.005 \\
\hline Surgery & $1871 * * *$ & $1876 * * *$ & $1670 * * *$ & $1672 * * *$ & $-0.012^{*}$ & $-0.012 *$ \\
\hline Admission at higher hospital & $4235 * * *$ & $4232 * * *$ & $4633^{* * *}$ & $4633^{* * *}$ & $0.177^{* * *}$ & $0.177 * * *$ \\
\hline Log length of stays & $3051 * * *$ & $3050 * * *$ & $2727 * * *$ & $2727 * * *$ & $-0.008^{*}$ & $-0.008^{*}$ \\
\hline NCMS coverage (\%) & $32.66 * * *$ & $24.40 * *$ & $13.89 *$ & 11.52 & $-0.003^{*}$ & -0.003 \\
\hline Year 2008 & $-1208 * *$ & $-970.6^{*}$ & $-1534 * * *$ & $-1460 * * *$ & -0.12 & $-0.140 * *$ \\
\hline Observations & 1226 & 1226 & 1226 & 1226 & 1227 & 1227 \\
\hline
\end{tabular}

Table 4. Regression results for financial protection for inpatient care. Marginal effects from GLM with log link and gamma family for spending and from GLM with logit link and binomial distribution for out of pocket share.

Notes: See Table 3. 


\begin{tabular}{lccc}
\hline & Total spending per visit (RMB) & Out-of-pocket spending per visit (RMB) & Out-of-pocket share (0-1) \\
\hline Log income & 18.24 & 17.12 & -0.000 \\
Rest days due to illness & $13.54^{* * *}$ & $12.16^{* * *}$ & $-0.001^{* *}$ \\
Visit THC (reference village & $228.0^{* * *}$ & $193.1^{* * *}$ & $-0.044^{* * *}$ \\
clinic) & $563.6^{* * *}$ & $527.0^{* * *}$ & $0.032^{* *}$ \\
Visit county hospital & $807.2^{* * *}$ & $744.3^{* * *}$ & $0.057^{* *}$ \\
Visit higher hospital & 11.74 & -17.85 & $-0.063^{* * *}$ \\
SPA (reference MSA) & 200.7 & 188.7 & $-0.082^{* *}$ \\
Year 2008 & 2164 & 2164 & 2182 \\
\hline Observations & & \\
\hline
\end{tabular}

Table 5. Regression results for financial protection for outpatient care. Marginal effects from GLM with log link and gamma family for spending and from GLM with logit link and binomial distribution for out of pocket share.

Notes: See Table 3. 


\begin{tabular}{lccc}
\hline & \multicolumn{1}{c}{ Total outpatient expenditure share (0-1) } & Total inpatient expenditure share (0-1) \\
\hline & OP model & Standardized ARR & Predicted ARR \\
\hline Log income & -0.0002 & $-0.0378^{* *}$ & $-0.0378^{* *}$ \\
SPA (reference MSA) & $0.0113^{* * *}$ & & \\
NCMS coverage (IP, \%) & & -0.0013 & 0.000 \\
Year 2008 & 0.0157 & -0.0046 & -0.0192 \\
\hline Observations & 5,680 & 5,680 & 5,680 \\
\hline
\end{tabular}

Table 6. Regression results for financial protection at the household level. Dependent variables are proportions of health expenditure in household expenditures (net of health expenditures) (0-1). Marginal effects from GLM with logit link and binomial distribution are shown.

Notes: The recall periods for total out- and inpatient are the last 4 weeks and 12 months respectively. All models include household-level covariates and village fixed effects. Standard errors are adjusted for clustering at county level. Significance level: *** at $1 \%, * *$ at $5 \%, *$ at $10 \%$. 


\begin{tabular}{|c|c|c|c|}
\hline Socio-economic covariates & Mean & Health status covariates & Mean \\
\hline Demographics & & $\begin{array}{l}\text { Self-perceived severity of sickness in the previous four } \\
\text { weeks }\end{array}$ & \\
\hline Male $16-29(1 / 0)$ & 0.141 & Mild (1/0) & 0.087 \\
\hline Male $30-44(1 / 0)$ & 0.159 & Moderate $(1 / 0)$ & 0.086 \\
\hline Male $45-64(1 / 0)$ & 0.15 & Serious $(1 / 0)$ & 0.061 \\
\hline Male $65+(1 / 0)$ & 0.042 & Type of sickness & \\
\hline Female $16-29(1 / 0)$ & 0.137 & Hypertension (1/0) & 0.031 \\
\hline Female $30-44(1 / 0)$ & 0.161 & Diseases of the respiratory system $(1 / 0)$ & 0.057 \\
\hline Female 45-64 (1/0) & 0.163 & Diseases of the digestive system $(1 / 0)$ & 0.023 \\
\hline Female $65+(1 / 0)$ & 0.047 & $\begin{array}{l}\text { Diseases of the musculoskeletal system and } \\
\text { connective tissue }(1 / 0)\end{array}$ & 0.047 \\
\hline Married (1/0) & 0.77 & Other diseases $(1 / 0)$ & 0.076 \\
\hline Household size (number) & 4.018 & Rest days due to sickness (days) & 0.914 \\
\hline Education & & Surgery $(1 / 0)$ & 0.021 \\
\hline Illiterate or semi-illiterate $(1 / 0)$ & 0.172 & Number of Chronic diseases in the last 12 months & \\
\hline Primary education $(1 / 0)$ & 0.212 & $1(1 / 0)$ & 0.17 \\
\hline Junior high school (1/0) & 0.461 & $2(1 / 0)$ & 0.03 \\
\hline Higher level education $(1 / 0)$ & 0.154 & 3 or more $(1 / 0)$ & 0.012 \\
\hline Occupation & & Types of Chronic diseases in the last 12 months & \\
\hline Farmer $(1 / 0)$ & 0.678 & Diseases of the nervous system $(1 / 0)$ & 0.011 \\
\hline Migrant worker(1/0) & 0.136 & Hypertension (1/0) & 0.043 \\
\hline Other occupation & 0.186 & Heart disease $(1 / 0)$ & 0.023 \\
\hline Total income per capita (RMB) & 6456 & Diseases of the respiratory system $(1 / 0)$ & 0.017 \\
\hline (standard deviation) & (9843) & Diseases of the digestive system $(1 / 0)$ & 0.027 \\
\hline Other health insurance scheme $(1 / 0)$ & 0.103 & $\begin{array}{l}\text { Diseases of the musculoskeletal system and } \\
\text { connective tissue (1/0) }\end{array}$ & 0.076 \\
\hline Observations & 26310 & Observations & 26310 \\
\hline
\end{tabular}

Appendix 1. Descriptive statistics of household covariates. 


\section{REFERENCES}

Angrist JD, Pischke J-S. 2009. Mostly Harmless Econometrics.. Princeton University Press: Princeton.

Babiarz KS, Miller G, Yi H, Zhang L, Rozelle S. 2010. New evidence on the impact of China's New Rural Cooperative Medical Scheme and its implications for rural primary healthcare: multivariate difference-in-difference analysis.BMJ 341(2): c5617.

Bertrand M, Duflo E, Mullainathan S. 2004. How much should we trust differences-in-differences estimates? Quarterly Journal of Economics 119(1): $249-275$

Brown PH, deBrauw A, Du Y. 2009. Understanding variation in the design of China's new cooperative medical system. The China Quarterly 198:304-329.

Brown PH, Theoharides C. 2009. Health-seeking behavior and hospital choice in China's new cooperative medical system. Health Economics 18 (S2): S47-S64.

Center for Health Statistics and Information. 2004. An Analysis Report of the National Health Survey in 2003 (in Chinese).

Center for Health Statistics and Information. 2009. An Analysis Report of the National Health Survey in 2008 (in Chinese).

Central Committee of the Communist Party and State Council. 2009. Opinions of the CPC Central Committee and the State Council on Deepening the Health Care System Reform. http://shs.ndrc.gov.cn/ygjd/ygwj/t20090408_271138.htm. 
Contoyannis P, Hurley J, Grootendorst P, Jeon SH, Tamblyn R. 2005. Estimating the price elasticity of expenditure for prescription drugs in the presence of non-linear price schedules: an illustration from Quebec, Canada. Health Economics 14(9): 909 - 923.

Hao Y, Wu Q, Zhang Z, Gao L, Ning N, Jiao M, Zakus D. 2010. The impact of different benefit packages of Medical Financial Assistance Scheme on health service utilization of poor population in Rural China. BMC Health Services Research 10(1): 170.

Hsiao W, Shaw P. 2007. Social Health Insurance for Developing Nations. World Bank: Washington, DC.

Hu S, Tang S, Liu Y, Zhao Y, Escobar ML, de Ferranti D. 2008. Reform of how health care is paid for in China: challenges and opportunities. Lancet 370:1846-1853.

Lei X, Lin W. 2009. The New Cooperative Medical Scheme in rural China: does more coverage mean more service and better health? Health Economics 18(S2): S25-S46.

Liu D, Tsegai D. 2011. The New Cooperative Medical Scheme (NCMS) and its implications for access to health care and medical expenditure: Evidence from rural China. ZEF-Discussion Papers on Development Policy No.155.

Liu X, Tang S, Yu B, Phuong NK, Yan F, Thien DD, Tolhurst R. 2012. Can rural health insurance improve equity in health care utilization? A comparison between China and Vietnam. International Journal for Equity in Health 11(1): 10. 
Manning WG, Mullahy J. 2001. Estimating log models: to transform or not to transform? Journal of Health Economics 20(4): 461-494.

Meng Q, Xu L, Zhang Y, Qian J, Cai M, Xin Y, Gao J, Xu K, Boerma JT, Barber SL. 2012. Trends in access to health services and financial protection in China between 2003 and 2011: a cross-sectional study. Lancet 379: 805-14.

Mihaylova B, Briggs A, O'Hagan A, Thompson SG. 2011. Review of statistical methods for analysing healthcare resources and costs. Health Economics20(8): 897-916.

Ministry of Health, P.R. China. 2010. China Health Statistical Yearbook. Peking Union Medical College Press: Beijing.

Ministry of Health, P.R. China. 2012. The Progress in 2011 and the focus in2012 of New Cooperative Medical Scheme (in Chinese).

Papke LE, Wooldridge JM. 1996. Econometric Methods for Fractional Response Variables with an Application to 401(K) Plan Participation Rates. Journal of Applied Econometrics 11(6): 619-632.

Qian D, Pong RW, Yin A, Nagarajan KV, Meng Q. 2009. Determinants of health care demand in poor, rural China: the case of Gansu Province. Health Policy and Planning 24(5):324-334.

Sun Q, Liu X, Meng Q, Tang S, Yu B, Tolhurst R. 2009. Evaluating the financial protection of patients with chronic disease by health insurance in rural China. International Journal for Equity in Health 8:42.

Wagstaff A, Yip W, LindelowM, Hsiao W. 2009a. China's health system and its reform: 
a review of recent studies. Health Economics 18(S2): S7-S23.

Wagstaff A, LindelowM, Gao J, Xu L, Qian J. 2009b. Extending health insurance to the rural population: An impact evaluation of China's new cooperative medical scheme. Journal of Health Economics 28(1): 1-19.

Wang H, Zhang L, Yip W, Hsiao W. 2011. An Experiment In Payment Reform For Doctors In Rural China Reduced Some Unnecessary Care But Did Not Lower Total Costs. Health Affairs 30(12): 2427-2436.

Wang HH, Huang S, Zhang L, Rozelle S, Yan Y. 2010. A comparison of rural and urban healthcare consumption and health insurance. China Agricultural Economic Review 2(2): 212-227.

Wooldridge JM. 2001. Applications of Generalized Method of Moments Estimation. Journal of Economic Perspectives 15(4):87-100.

Wooldridge JM. 2002. Econometric Analysis of Cross Section and Panel Data. MIT Press: Cambridge.

World Health Organization.2007.International Statistical Classification of Diseases and Related Health Problems (10th Revision). Available from:http://apps.who.int/classifications/apps/icd/icd10online/.

Yan F, Raven J, Wang W, Tolhurst R, Zhu K, Yu B, Collins C. 2011. Management capacity and health insurance: the case of the New Cooperative Medical Scheme in six counties in rural China. The International Journal of Health Planning and Management 26(4):357-378. 
Yip W, Hsiao W. 2008. The Chinese health system at a crossroads. Health Affairs (Millwood) 27(2): 460-468.

Yip W, Hsiao W. 2009. China's health care reform: A tentative assessment. China Economic Review 20: 613-619.

Yip W, Hsiao W, Meng Q, Chen W, Sun X. 2010. Realignment of incentives for health-care providers in China. Lancet 375: 1120-30.

Yip W, Hsiao W, Chen W, Hu S, Ma J, Maynard A. 2012. Early appraisal of China's huge and complex health-care reforms. Lancet 379: 833-42.

Yip W, Wagstaff A, Hsiao W. 2009. Economic analysis of China's health care system: turning a new page. Health Economics 18(S2): S3-S6.

You X, Kobayashi Y. 2009. The new cooperative medical scheme in China. Health Policy 91(1): 1-9.

YuB, Meng Q, Collins C, Tolhurst R, Tang S, Yan F, Bogg L, Liu X. 2010. How does the New Cooperative Medical Scheme influence health service utilization? A study in two provinces in rural China.BMC Health Services Research 10(1): 116.

Zhang L, Cheng X, Liu X, Zhu K, Tang S, Bogg L, Dobberschuetz K, Tolhurst R. 2010. Balancing the funds in the New Cooperative Medical Scheme in rural China: determinants and influencing factors in two provinces. The International Journal of Health Planning and Management 25(2): 96-118.

Zhong H. 2011. Effect of patient reimbursement method on health-care utilization: evidence from China.Health Economics20: 1312-1329. 
Zhou Z, Gao J, Xue Q, Yang X, Yan J. 2009. Effects of Rural Mutual Health Care on outpatient service utilization in Chinese village medical institutions: evidence from panel data.Health Economics 18(S2): S129-S136.

Zhou Z, Gao J, Fox A, Rao K, Xu K, Xu L, Zhang Y. 2011.Measuring the equity of inpatient utilization in Chinese rural areas. BMC Health Services Research11: 201. 